\title{
Chemical Processes in Astrophysical Radiation Fields
}

ORNL/CP-96202

P. C. Stancil

CONF-980417- -

Physics Division, Oak Ridge National Laboratory, P.O. Box 2008, Oak Ridge, TN 37831-6372

\author{
A. Dalgarno
}

Harvard-Smithsonian Center for Astrophysics, 60 Garden St., Cambridge, MA 02138

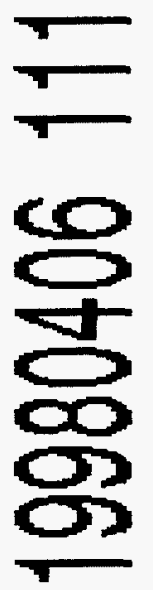

\begin{abstract}
The effects of stimulated photon emission on chemical processes in a radiation field are considered and their influence on the chemistry of the early universe and other astrophysical environments is investigated. Spontaneous and stimulated radiative attachment rate coefficients for $\mathrm{H}^{-}, \mathrm{Li}^{-}$and $\mathrm{C}^{-}$are presented.
\end{abstract}

AECEIVED

\section{INTRODUCTION}

In astrophysical environments lacking dust, molecules can only be formed through gasphase chemistry. Two of the primary mechanisms involve radiative processes: (i) the radiative association of two neutral species (or a neutral and an ion)

$$
X+Y \rightarrow X Y+h \nu
$$

and (ii) the negative-ion sequence of radiative attachment

$$
X+e^{-} \rightarrow X^{-}+h \nu
$$

followed by associative detachment

$$
X^{-}+Y \rightarrow X Y+e^{-}
$$
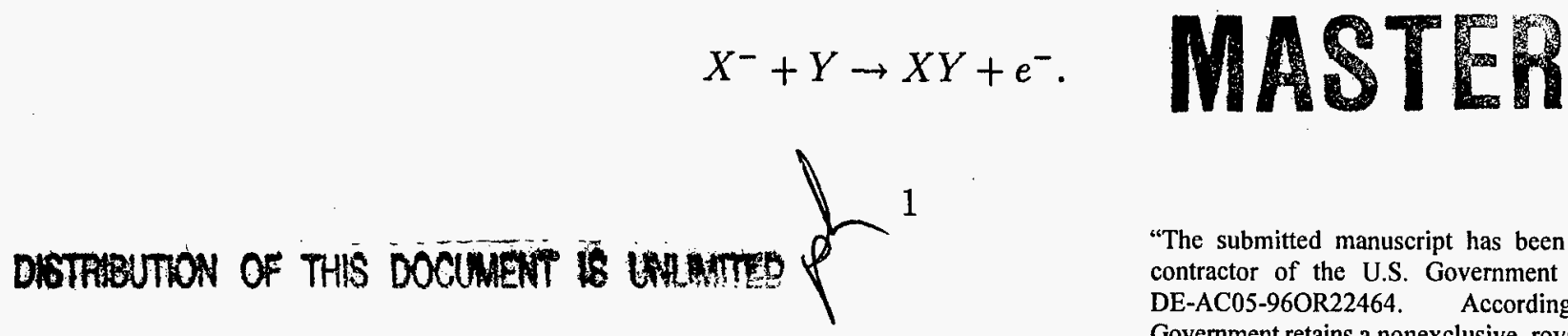
Until recently, only spontaneous photon emission has been considered in investigations of reactions (1) and (2). If these processes occur in an environment with an intense infrared radiation field there is a possibility that the reaction rate coefficients can be enhanced by stimulated effects:

$$
X+Y+h \nu_{\mathrm{b}} \rightarrow X Y+h \nu+h \nu_{\mathrm{b}}
$$

and

$$
X+e^{-}+h \nu_{\mathrm{b}} \rightarrow X^{-}+h \nu+h \nu_{\mathrm{b}} .
$$

In the above processes $\nu$ is the frequency of the stabilizing spontaneous emission photon and $\nu_{\mathrm{b}}$ the frequency of the radiation field.

Dubrovich $^{1}$ has suggested that the stimulated mechanism may operate in the recombination era of the early universe, enhancing the abundance of $\mathrm{LiH}$, due to the cosmic background radiation (CBR) field. Stancil and Dalgarno ${ }^{2}$ carried out explicit calculations for the formation of $\mathrm{LiH}$ via reaction (4). They found that indeed the rate coefficients are considerably enhanced; however other factors of the environment and chemistry conspired to keep the stimulated effects from playing a significant role in the formation of $\mathrm{LiH}$ in the early universe.

In this Discussion, we extend the work of Stancil and Dalgarno ${ }^{2}$ to the radiative attachment process (5) and investigate the influence of stimulated effects in the early universe, supernova ejecta, and other astrophysical environments.

\section{THEORY}

The extension of the theory of radiative association to include stimulated effects was outlined by Stancil and Dalgarno ${ }^{2}$. Essentially, the partial cross section for a transition into a bound vibrational-rotational level of the resulting molecule is enhanced by the factor

$$
\frac{1}{1-\exp \left(-h \nu / k T_{\mathbf{b}}\right)}
$$




\section{DISCLAIMER}

This report was prepared as an account of work sponsored by an agency of the United States Government. Neither the United States Government nor any agency thereof, nor any of their employees, makes any warranty, express or implied, or assumes any legal liability or responsibility for the accuracy, completeness, or usefulness of any information, apparatus, product, or process disclosed, or represents that its use would not infringe privately owned rights. Reference herein to any specific commercial product, process, or service by trade name, trademark, manufacturer, or otherwise does not necessarily constitute or imply its endorsement, recommendation, or favoring by the United States Government or any agency thereof. The views and opinions of authors expressed herein do not necessarily state or reflect those of the United States Government or any agency thereof. 
for a blackbody radiation field characterized by the temperature $T_{\mathrm{b}}$. The enhancement increases with $T_{\mathrm{b}}$ or decreasing $\nu$. The theory has been applied to the formation of HD (Stancil and Dalgarno $\left.{ }^{3}\right), \mathrm{HeH}^{+}\left(\right.$Zygelman et al. $\left.{ }^{4}\right)$, and $\mathrm{LiH}\left(\right.$ Stancil and Dalgarno ${ }^{2}$ ).

The theory of stimulated radiative attachment is obtained in an analogous way. The negative ion $X^{-}$is created by reactions (2) and (5) and destroyed by the photodetachment process

$$
X^{-}+h \nu \rightarrow X+e^{-}
$$

where the photon energy $E_{\nu}=h \nu=E+E_{0}, E_{0}$ is the electron affinity of $X$, and $E$ is the relative translational energy of the products $X$ and $e^{-}$.

Cross sections for reactions (2) and (7) are related through detailed balance $e^{5,6}$

$$
\sigma_{7}=\left(\frac{\mu c v}{h \nu}\right)^{2} \frac{g_{0}}{g_{-}} \sigma_{2}
$$

where $g_{0}$ and $g_{-}$are the statistical weights of the atom and negative ion, respectively, $\mu$ is the reduced mass, and $v$ the relative collision velocity. Equation (8) can be rewritten as

$$
\sigma_{2}(E)=\frac{\left(E+E_{0}\right)^{2}}{2 \mu c^{2} E} \frac{g_{-}}{g_{0}} \sigma_{7}(E) .
$$

For a blackbody radiation field, the photon density per unit frequency interval is

$$
\rho\left(\nu_{\mathrm{b}}, T_{\mathrm{b}}\right)=\frac{8 \pi h \nu_{\mathrm{b}}^{3}}{c^{3}} \frac{1}{\exp \left(h \nu_{\mathrm{b}} / k T_{\mathrm{b}}\right)-1}
$$

and the stimulated cross section is related to the spontaneous cross section by

$$
\sigma_{5}(E)=\frac{c^{3}}{8 \pi h \nu^{3}} \sigma_{2}(E)
$$

Combining equations (9), (10) and (11) gives the total, spontaneous plus stimulated, radiative attachment cross section

$$
\begin{aligned}
\sigma_{2+5}\left(E, T_{\mathrm{b}}\right) & =\sigma_{2}(E)+\sigma_{5}(E) \rho\left(\nu, T_{\mathrm{b}}\right) \\
& =\frac{\left(E+E_{0}\right)^{2}}{2 \mu c^{2} E} \frac{g_{-}}{g_{0}} \frac{\sigma_{7}(E)}{1-\exp \left(-h \nu / k T_{\mathrm{b}}\right)} .
\end{aligned}
$$


The total radiative attachment rate coefficient for $T_{\mathrm{b}}$ and collision temperature $T_{\mathrm{c}}$ can be written

$$
\begin{aligned}
\alpha_{2+5}\left(T_{\mathrm{c}}, T_{\mathrm{b}}\right) & =\frac{g_{-}}{g_{0}}\left(\frac{2}{c^{4} \pi}\right)^{1 / 2}\left(\frac{1}{\mu k T_{\mathrm{c}}}\right)^{3 / 2} \exp \left(E_{0} / k T_{\mathrm{c}}\right) \\
& \times \int_{E_{0}}^{\infty} \frac{E_{\nu}^{2} \sigma_{7}\left(E_{\nu}\right) \exp \left(-E_{\nu} / k T_{\mathrm{c}}\right)}{1-\exp \left(-E_{\nu} / k T_{\mathrm{b}}\right)} d E_{\nu} .
\end{aligned}
$$

Therefore, given the photodetachment cross section, the electron affinity, and the statistical weights, rate coefficients can be readily obtained with the application of equation (13).

\section{RESULTS AND DISCUSSION}

\section{A. $\mathrm{H}^{-}$}

Fig. 1 presents the total radiative attachment rate coefficients for the formation of $\mathrm{H}^{-}$ obtained with the reliable photodetachment cross sections of Sadeghpour, Dalgarno and Stancil ${ }^{7}$. The cross section consists of a combination of new R-matrix calculations and previous experimental and theoretical results and its accuracy is controlled by ensuring that a number of oscillator strength sum-rules are satisfied. The spontaneous radiative attachment $\left(T_{\mathrm{b}}=0 \mathrm{~K}\right)$ results are in good accord with the previous calculations of Dalgarno and Kingston ${ }^{8}$, however the rate coefficients used by Rawlings et al. ${ }^{9}$ are in serious disagreement. As the rate coefficients of Rawlings et al..$^{9}$ have been adopted in the astrochemistry database of Millar et $a l .{ }^{10}$, the abundance of $\mathrm{H}^{-}$may have been underestimated in some astrophysical models. Consequently, $\mathrm{H}_{2}$ may have also been underestimated as it is primarily formed by

$$
\mathrm{H}^{-}+\mathrm{H} \rightarrow \mathrm{H}_{2}+e^{-}
$$

in dust-free and relatively cool environments such as the early universe for redshifts $z<100$.

The effect of stimulated emission is negligible for $T_{\mathrm{b}}<3000 \mathrm{~K}$, but very pronounced when $T_{\mathrm{b}}>10,000 \mathrm{~K}$, for all considered collision temperatures. 
Total radiative attachment rate coefficients for the formation of $\mathrm{Li}^{-}$are displayed in Fig. 2. The rate coefficients were calculated using the R-matrix photodetachment results of Ramsbottom et al. ${ }^{11}$. We are unaware of any previous calculations except for the rate coefficients adopted by Stancil, Lepp and Dalgarno ${ }^{12}$ for a model of the early universe lithium chemistry. Their adopted fit of $1.85 \times 10^{-15}\left(T_{c} / 300\right)^{0.62} \exp \left(-T_{c} / 9300\right) \mathrm{cm}^{3} / \mathrm{s}$ should be replaced by the fit to the present spontaneous $\left(T_{\mathrm{b}}=0 \mathrm{~K}\right)$ radiative attachment rate coefficients: $1.53 \times 10^{-15}\left(T_{\mathrm{c}} / 300\right)^{0.92} \exp \left(-T_{\mathrm{c}} / 4560\right) \mathrm{cm}^{3} / \mathrm{s}$. The temperature dependence is similar to that of $\mathrm{H}^{-}$. Since both species involve the photodetachment of a $s$-electron resulting in a $p$-wave final state, their photodetachment cross section near threshold obeys the Wigner law, $\sigma_{7} \propto E^{3 / 2}$.

As for $\mathrm{H}^{-}$, the enhancement due to stimulated attachment is small for $T_{\mathrm{b}}<3000 \mathrm{~K}$, but increases significantly for $T_{\mathrm{b}}>10,000 \mathrm{~K}$.

\section{C. $\mathbf{C}^{-}$}

Total radiative attachment rate coefficients for the formation of ground state $\mathrm{C}^{-}\left({ }^{4} S^{\circ}\right)$ are presented in Fig. 3. The calculations used the measured photodetachment cross section of Seman and Branscomb ${ }^{13}$ for $E_{\nu}$ between 1.5 and $2.04 \mathrm{eV}$, the R-matrix calculations of Ramsbottom et al. ${ }^{14}$ for $E_{\nu}>2.04 \mathrm{eV}$, and a fit to a Wigner threshold law for $E_{\nu}<1.5$ $\mathrm{eV}$. We are unaware of any previous calculations of the spontaneous radiative attachment rate coefficients, but a temperature independent value of $3.0 \times 10^{-15}$ was adopted by Millar et al..$^{10}$. The Millar et al. ${ }^{10}$ value is about $30 \%$ larger than the current results suggesting that some astrochemistry models may have slightly overestimated the $\mathrm{C}^{-}$abundance. The fit $2.25 \times 10^{-15} \exp \left(-T_{\mathrm{c}} / 9000\right) \mathrm{cm}^{3} / \mathrm{s}$ for the present spontaneous $\left(T_{\mathrm{b}}=0 \mathrm{~K}\right)$ radiative attachment rate coefficients should be used for $T_{\mathrm{c}}<1000 \mathrm{~K}$.

Results for the formation of the metastable $\mathrm{C}^{-}\left({ }^{2} D^{\circ}\right)$ are presented in Fig. 4. The rate co- 
efficients were obtained using the R-matrix photodetachment cross sections of Ramsbottom et al. ${ }^{14}$ for $E_{\nu}>0.067 \mathrm{eV}$ and a fit to a Wigner threshold law for smaller energies.

Similar to $\mathrm{H}^{-}$and $\mathrm{Li}^{-}$, stimulated effects only become important for $T_{\mathrm{b}}>10,000 \mathrm{~K}$ for the ground state, but for the metastable state the enhancements can be significant to $T_{\mathrm{b}}$ as low as $\sim 500 \mathrm{~K}$. As the electron affinity decreases $\left({ }^{4} S^{o}: 1.263 \mathrm{eV} ;{ }^{2} D^{o}: 0.035 \mathrm{eV}\right)$, the stimulated enhancement increases, which is related to the appearance of $E_{\nu}=E+E_{0}$ in the enhancement factor in equation (13). To further visualize this behavior, Fig. 5 displays the spontaneous and total radiative attachment rate coefficients for a model negative ion with arbitrary electron affinity.

\section{ASTROPHYSICAL APPLICATIONS}

\section{A. The Early Universe}

The rationale for investigating stimulated effects on molecular formation was motivated by studies of chemistry in the early universe. The early expanding universe is embedded in the CBR field left over from the big bang with a temperature that decreases with decreasing redshift $z ; T_{\mathrm{b}}=2.726(1+z) \mathrm{K}$. The temperature of the gas $T_{\mathrm{c}}$ is coupled to that of the CBR field until the beginning of the recombination era $(z \sim 1300)$, after which $T_{\mathrm{b}}>T_{\mathrm{c}} \propto(1+z)^{2}$. The CBR field is sufficiently intense to prevent significant formation of negative ions until after $z \sim 200$ due to the high efficiency of photodetachment. Since by then $T_{\mathrm{b}}$ had fallen to less than $600 \mathrm{~K}$, it is clear from Figs. 1 and 2 that stimulated radiative attachment played an insignificant role in the formation of $\mathrm{H}^{-}$and $\mathrm{Li}^{-}$in the early universe. Additionally, there is no increase in the abundances of $\mathrm{H}_{2}$ and $\mathrm{LiH}$ due to associative detachment, process (3), following stimulated radiative attachment.

Molecules do not begin to form in significant abundances until $z<500$. At larger redshifts they are primarily destroyed by photodissociation. The effects of stimulated emission are generally larger for radiative association than radiative attachment for a comparable $T_{\mathrm{b}}$ 
and even appreciable for $T_{\mathrm{b}}$ as low as $500 \mathrm{~K}^{2-4}$. This is because the energy of the stabilizing photons can be much smaller $\left(E_{\nu}<0.2 \mathrm{eV}\right)$ for transitions into high-lying bound rotationalvibrational levels than the minimum photon energy for a negative ion, the electron affinity. Since $T_{\mathrm{b}} \sim 1300 \mathrm{~K}$ at $z=500$, it is suggestive that stimulated radiative association may contribute to molecule formation. Stancil and Dalgarno ${ }^{2}$ and Zygelman, Stancil and Dalgarno find that the abundances of $\mathrm{LiH}$ and $\mathrm{HeH}^{+}$are enhanced by $\sim 25 \%$. However, this is only marginally significant considering that the fractional abundances (with respect to hydrogen nuclei) are $\sim 10^{-17}$ and $\sim 10^{-15}$ for $\mathrm{LiH}$ and $\mathrm{HeH}^{+}$, respectively ${ }^{12,15}$.

\section{B. Supernova Ejecta}

While the environmental conditions of a supernova $(\mathrm{SN})$ appear to be hostile to the formation of molecules, $\mathrm{CO}$ and $\mathrm{SiO}$ were observed in the ejecta of the Type II object SN $1987 \mathrm{~A}^{16}$. The visible and infrared spectrum of SN 1987A consisted of photospheric-like emission from the central region with an effective temperature of $\sim 5000 \mathrm{~K}$ and an infrared excess whose intensity increased with relative to the photosphere. The infrared excess is believed to be due to dust which probably formed in the same region as the molecules, the molecules acting as the dust nucleation sites.

The chemistry of CO, prior to the production of dust, was described by Lepp, Dalgarno and $\mathrm{McCray}{ }^{17}$, the primary reactions being radiative association

$$
\mathrm{C}+\mathrm{O} \rightarrow \mathrm{CO}+h \nu
$$

and the negative ion sequence

$$
\begin{aligned}
& \mathrm{C}+e^{-} \rightarrow \mathrm{C}^{-}+h \nu, \\
& \mathrm{C}^{-}+\mathrm{O} \rightarrow \mathrm{CO}+e^{-}
\end{aligned}
$$

Stimulated effects are probably small for reaction (15) since CO has a large dissociation energy ( $11 \mathrm{eV}$ compared to only 2.0 and $2.4 \mathrm{eV}$ for $\mathrm{HeH}^{+}$and $\mathrm{LiH}$, respectively). Stimulated radiative attachment may play a role as suggested by Figs. 3 and 4 . 
Wooden et $a l .^{18}$ have modelled the photosphere and dust emission by fitting the spectra of SN 1987 A to scaled Planck functions. Using their fits an estimate of a blackbody dilution factor, consisting of geometric dilution and departure from a blackbody intensity, can be made. At 260 days, the dilution factor from the photosphere to the $\mathrm{C}-\mathrm{O}$ region of the ejecta is about $2 \times 10^{-3}$. Given the dilution factor, the photospheric temperature of $5000 \mathrm{~K}$, and the $\mathrm{C}-\mathrm{O}$ gas temperature of $2000 \mathrm{~K}^{19}$, the total $\mathrm{C}^{-}\left({ }^{4} S^{\circ}\right.$ and $\left.{ }^{2} D^{\circ}\right)$ radiative attachment rate is enhanced by $<0.05 \%$. As the dust is formed in the $\mathrm{C}-\mathrm{O}$ region, we take its dilution factor to be of the order of unity. Given the dust temperature of $700 \mathrm{~K}$, the formation of $\mathrm{C}^{-}$is enhanced by $0.5 \%$. While this suggests that $\mathrm{CO}$ formation is not affected by stimulated effects due to the continuum radiation from the supernova, line emission from the fundamental $\mathrm{CO}$ band $(\sim 4.5 \mu \mathrm{m})$ may provide some enhancement as the line-to-continuum flux ratio is $\sim 10$.

The situation is similar for $\mathrm{H}^{-}$, which considering the photospheric and dust radiation, is only enhanced by $0.03 \%$. There is a possibility that the abundance of $\mathrm{Fe}^{-}$may be affected by stimulated processes since the iron core of the ejecta is in closer proximity to the photosphere and its electron affinity $(0.16 \mathrm{eV})$ is smaller than $\mathrm{H}^{--}$or $\mathrm{C}^{-}$. Fig. 5 shows that enhancements due to stimulated radiative attachment increase with decreasing electron affinity. If the total dilution factor between the photosphere and iron zone is greater than $4 \times 10^{-3}$ then the enhancement in the $\mathrm{Fe}^{-}$formation is $>1 \%$.

Two emission lines in SN 1987A have been tentatively identified by Miller et al. ${ }^{20}$ to be due to $\mathrm{HeH}^{+}$. It is primarily formed in the hydrogen envelope of the ejecta by the radiative association process

$$
\mathrm{He}^{+}+\mathrm{H} \rightarrow \mathrm{HeH}^{+}+h \nu
$$

but

$$
\mathrm{He}+\mathrm{H}^{+} \rightarrow \mathrm{HeH}^{+}+h \nu
$$

may contribute. Zygelman et $a{ }^{4}{ }^{4}$ have calculated the rate coefficients for reaction (19) and for the stimulated radiative association reaction 


$$
\mathrm{He}+\mathrm{H}^{+}+h \nu_{\mathrm{b}} \rightarrow \mathrm{HeH}^{+}+h \nu+h \nu_{\mathrm{b}}
$$

The analogous stimulated process for reaction (18) is probably negligible except for large $T_{\mathrm{b}}$ as the minimum stabilizing photon energy is $10.99 \mathrm{eV}$. Similar to the $\mathrm{C}-\mathrm{O}$ zone, the dilution factor for the hydrogen envelope is about $8 \times 10^{-4}$ from the photosphere and 0.6 from the dust at 260 days. Stimulated association enhances the formation of $\mathrm{HeH}^{+}$by $0.2 \%$ and $9 \%$ due to the photosphere and dust, respectively compared to reaction (19). However, reaction (18) produces about $\sim 85 \%$ of the $\mathrm{HeH}^{+}$(assuming a hydrogen ionization fraction of 0.01 ), so that the total $\mathrm{HeH}^{+}$production is only enhanced by $1.5 \%$.

\section{Other Environments}

Dubrovich and Lipovka ${ }^{21}$ have studied the formation of $\mathrm{HeH}^{+}$in high redshift Lyman $\alpha$ clouds irradiated by a background quasar and suggest that $\mathrm{HeH}^{+}$could be detected with millimeter observations. However, the $\mathrm{HeH}^{+}$abundance produced by spontaneous radiative association, reactions (18) and (19), is probably not much larger than in the intergalactic medium (comparable to the early universe value of $10^{-15}$ ) and Dubrovich ${ }^{1}$ has suggested that $\mathrm{HeH}^{+}$could be enhanced by reaction (20) due to the quasar radiation. Their models suggest that the $\mathrm{HeH}^{+}$abundance is significant if the Lyman $\alpha$ cloud is $>10^{23} \mathrm{~cm}$ from the quasar. Assuming the quasar to radiate as a blackbody with a radius of $10^{19} \mathrm{~cm}$, one obtains a geometric dilution factor of $10^{-8}$ suggesting stimulated effects to be negligible.

The situation is similar in a planetary nebulae, but more severe. For the white dwarf effective temperature of $175,000 \mathrm{~K}$ for NGC 7027 , molecules begin to form for distances greater than $10^{16} \mathrm{~cm}$ from the central $\mathrm{star}^{22}$, corresponding to a dilution factor of $10^{-14}$.

One possibility where stimulated effects may play a role is in quasar broad-line clouds. Kallman, Lepp and Giovannoni ${ }^{23}$ have found that the fractional abundance of $\mathrm{H}_{2}$ can reach 0.5 at a depth of $10^{14} \mathrm{~cm}$ into the cloud. Given that this distance is much smaller than the radius of the quasar, there is no geometric dilution. For a quasar effective temperature of $50,000 \mathrm{~K}$, this implies that the abundances of $\mathrm{H}^{-}$and $\mathrm{HeH}^{+}$could be enhanced by factors 
of up to 5 and 20, respectively. However, a detailed model of a broad-line cloud will be necessary to test this prediction.

\section{SUMMARY}

In this Discussion recent work on stimulated emission effects in the formation of diatomic molecules has been extended to stimulated radiative attachment to form negative atomic ions. Rate coefficients for the production of $\mathrm{H}^{-}, \mathrm{Li}^{-}$and $\mathrm{C}^{-}$have been given for a range of collision and radiation temperatures. Corrections to currently adopted spontaneous radiative attachment rate coefficients have been indicated.

Radiative association and attachment are significantly enhanced by stimulated emission due to a blackbody radiation field, with the enhancement to association occurring at somewhat smaller radiation temperatures than for attachment. However, in the astrophysical environments considered thus far, this enhancement is generally negated by other mitigating circumstances, geometric dilution being the primary culprit. Geometric dilution appears to be particularly severe in planetary nebulae and quasar irradiated Lyman $\alpha$ clouds. Minor enhancements in molecule formation may be found in Type II supernova ejecta as the dilution is more moderate. While dilution is not a concern in the early universe, only modest enhancements are obtained for molecules since large radiation temperatures are very effective at removing molecules and negative ions by photo-destruction processes. As quasar broad-line clouds are thought to be nearly fully molecular at their core and are in close proximity to the intense quasar radiation, they may be the prototypical environment for stimulated formation effects.

\section{ACKNOWLEDGMENTS}

We thank H. R. Sadeghpour for providing the $\mathrm{H}^{-}$photodetachment cross section. A. D. acknowledges support from NSF grant AST 95-31790. The work of P. C. S. was performed as a Eugene P. Wigner Fellow and staff member at the Oak Ridge National Laboratory, 
managed by Lockheed Martin Energy Research Corp for the U. S. Department of Energy under Contract DE-AC05-96OR22464. 


\section{REFERENCES}

${ }^{1}$ V. K. Dubrovich, 1996, private communication.

${ }^{2}$ P. C. Stancil and A. Dalgarno, Astrophys. J., 1997a, 479, 543.

${ }^{3}$ P. C. Stancil and A. Dalgarno, Astrophys. J., 1997b, 490, 76.

${ }^{4}$ B. Zygelman, P. C. Stancil and A. Dalgarno, 1998, in preparation.

${ }^{5}$ H. S. W. Massey, Negative Ions, Cambridge University Press, Cambridge, 1950.

${ }^{6}$ L. M. Branscomb, in Atomic and Molecular Processes, ed. D. R. Bates, Academic Press, New York, 1962.

${ }^{7}$ H. R. Sadeghpour, A. Dalgarno and P. C. Stancil, 1998, in preparation.

${ }^{8}$ A. Dalgarno and A. E. Kingston, Observatory, 1963, 83, 39.

${ }^{9}$ J. M. C. Rawlings, D. A. Williams and J. Canto, Mon. Not. Roy. Astron. Soc., 1988, 230, 695.

${ }^{10}$ T. J. Millar, P. R. A. Farquhar and K. Willacy, Astron. Astrophys. Suppl., 1997, 121, 139.

${ }^{11}$ C. A. Ramsbottom, K. L. Bell and K. A. Berrington, J. Phys. B: At. Mol. Opt. Phys., $1994,27,2905$.

12 P. C. Stancil, S. Lepp and A. Dalgarno, Astrophys. J., 1996, 458, 401.

${ }^{13}$ M. L. Seman and L. M. Branscomb, Phys. Rev., 1962, 125, 1602.

${ }^{14}$ C. A. Ramsbottom, K. L. Bell and K. A. Berrington, J. Phys. B: At. Mol. Opt. Phys., $1993,26,4399$.

${ }^{15}$ P. C. Stancil, S. Lepp and A. Dalgarno, 1998, in preparation.

${ }^{16}$ W. P. S. Meikle, et al., Mon. Not. R. Astron. Soc., 1989, 238, 193.

${ }^{17}$ S. Lepp, A. Dalgarno and R. McCray, Astrophys. J, 1990, 358, 262. 
${ }^{18}$ D. H. Wooden, et al., Astrophys. J. Suppl., 1993, 88, 477.

${ }^{19}$ W. Liu and A. Dalgarno, Astrophys. J., 1995, 454, 472.

${ }^{20}$ S. Miller, J. Tennyson, S. Lepp and A. Dalgarno, Nature, 1992, 355, 420.

${ }^{21}$ V. K. Dubrovich, V. K. and A. A. Lipovka, Astron. Astrophys., 1995, 296, 309.

${ }^{22}$ C. Cecchi-Pestellini and A. Dalgarno, Astrophys. J., 1993, 413, 611.

${ }^{23}$ T. Kallman, S. Lepp and P. Giovannoni, Astrophys. J., 1987, 321, 907. 


\section{FIGURES}

FIG. 1. Rate coefficients for the formation of $\mathrm{H}^{-}$by spontaneous and stimulated radiative attachment in a blackbody radiation field of temperature $T_{\mathrm{b}}$. Current results (thick lines), $T_{\mathrm{b}}=0$, $3000,5000,10000,12500,15000,20000,30000,40000,50000 \mathrm{~K}$ (bottom to top). Previous results for $T_{\mathrm{b}}=0$ : Dalgarno and Kingston ${ }^{8}$ (thin solid line) and Rawlings et al. ${ }^{9}$ (thin dot-dash line).

FIG. 2. Rate coefficients for the formation of $\mathrm{Li}^{-}$by spontaneous and stimulated radiative attachment in a blackbody radiation field of temperature $T_{\mathrm{b}}$. Current results (thick lines), $T_{\mathrm{b}}=0$, $3000,5000,7000,10000,12500,15000,20000,30000,40000,50000 \mathrm{~K}$ (bottom to top). The thin solid line is the adopted spontaneous, $T_{\mathrm{b}}=0 \mathrm{~K}$, rate coefficient of Stancil et al. ${ }^{12}$

FIG. 3. Rate coefficients for the formation of the ground state $\mathrm{C}^{-}\left({ }^{4} S^{\circ}\right)$ by spontaneous and stimulated radiative attachment in a blackbody radiation field of temperature $T_{\mathrm{b}}$. Current results (thick lines), $T_{\mathrm{b}}=0,5000,7000,10000,12500,15000,20000,30000,40000,50000 \mathrm{~K}$ (bottom to top). The thin solid line is the adopted spontaneous, $T_{\mathrm{b}}=0 \mathrm{~K}$, rate coefficient of Millar et al. ${ }^{10}$

FIG. 4. Rate coefficients for the formation of the metastable state $\mathrm{C}^{-}\left({ }^{2} D^{\circ}\right)$ by spontaneous and stimulated radiative attachment in a blackbody radiation field of temperature $T_{\mathrm{b}}$. Current results (thick lines), $T_{\mathrm{b}}=0,500,1000,2000,3000,5000,7000,10000,12500,15000,20000,30000$, $40000,50000 \mathrm{~K}$ (bottom to top). The thin solid line is the adopted spontaneous, $T_{\mathrm{b}}=0 \mathrm{~K}$, rate coefficient of Millar et al. ${ }^{10}$ for the ground state.

FIG. 5. Radiative attachment rate coefficients for a model negative ion with arbitrary electron affinity for $T_{\mathrm{c}}=2000 \mathrm{~K}$ : spontaneous attachment, $T_{\mathrm{b}}=0 \mathrm{~K}$ (solid line); spontaneous and stimulated attachment, $T_{\mathrm{b}}=5000 \mathrm{~K}$ (dotted line). The electron affinities of the first thirty elements are given along the top of the figure (crosses). Explicit results from Figs. 1 through 4 are given for comparison: $T_{\mathrm{b}}=0 \mathrm{~K}$ (open squares); $T_{\mathrm{b}}=5000 \mathrm{~K}$ (filled diamonds). The statistical weights are set to unity in all cases. 


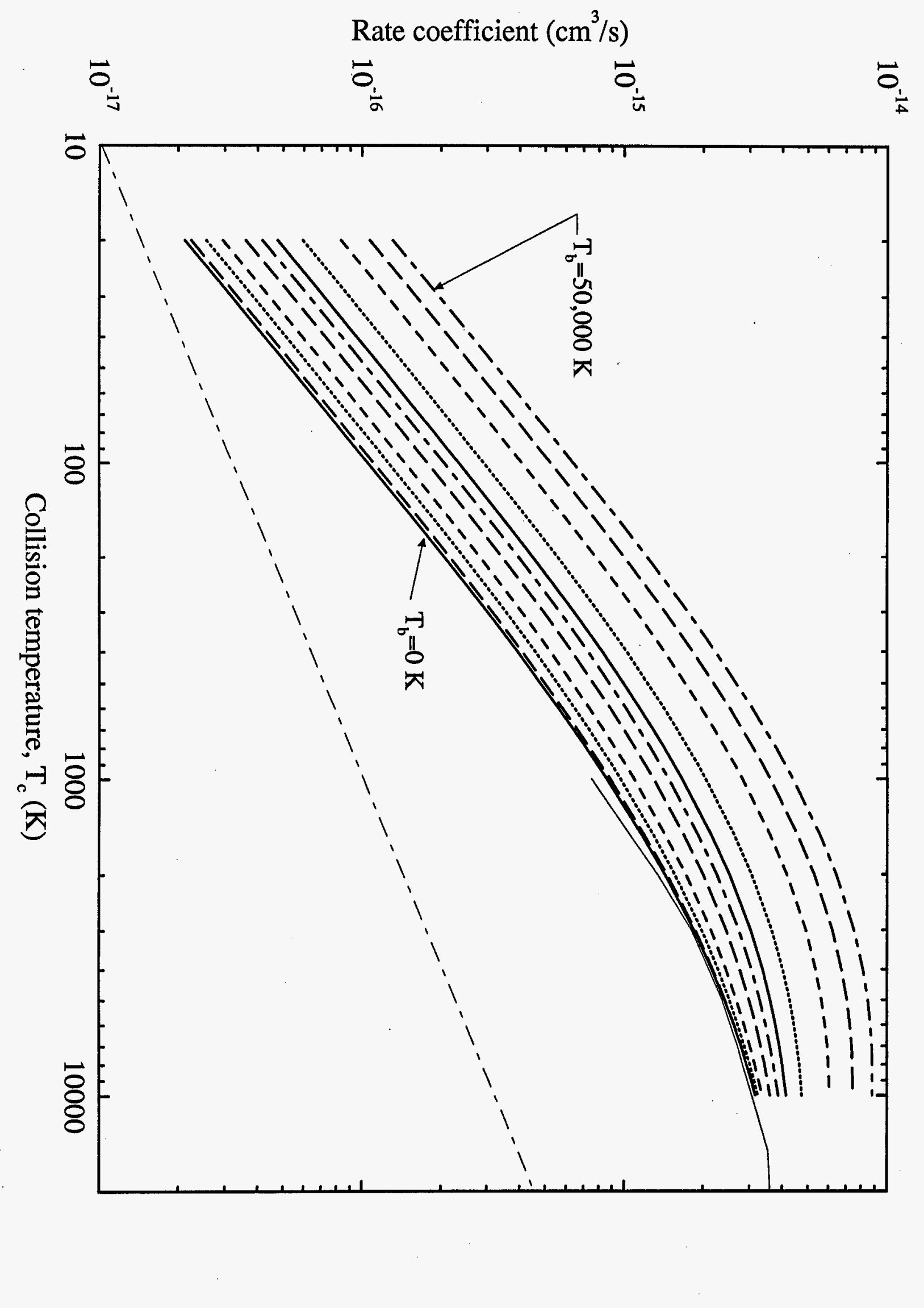




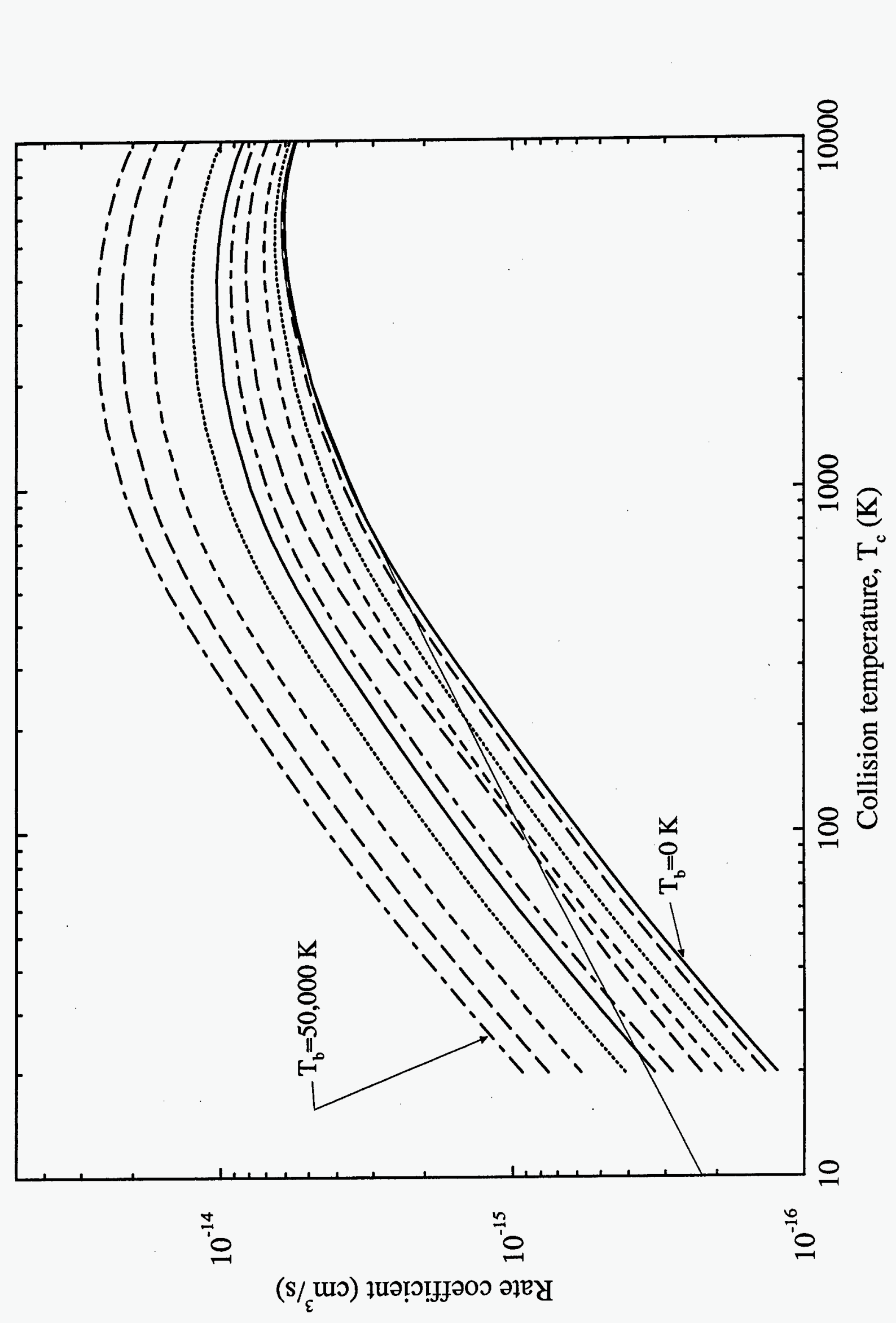




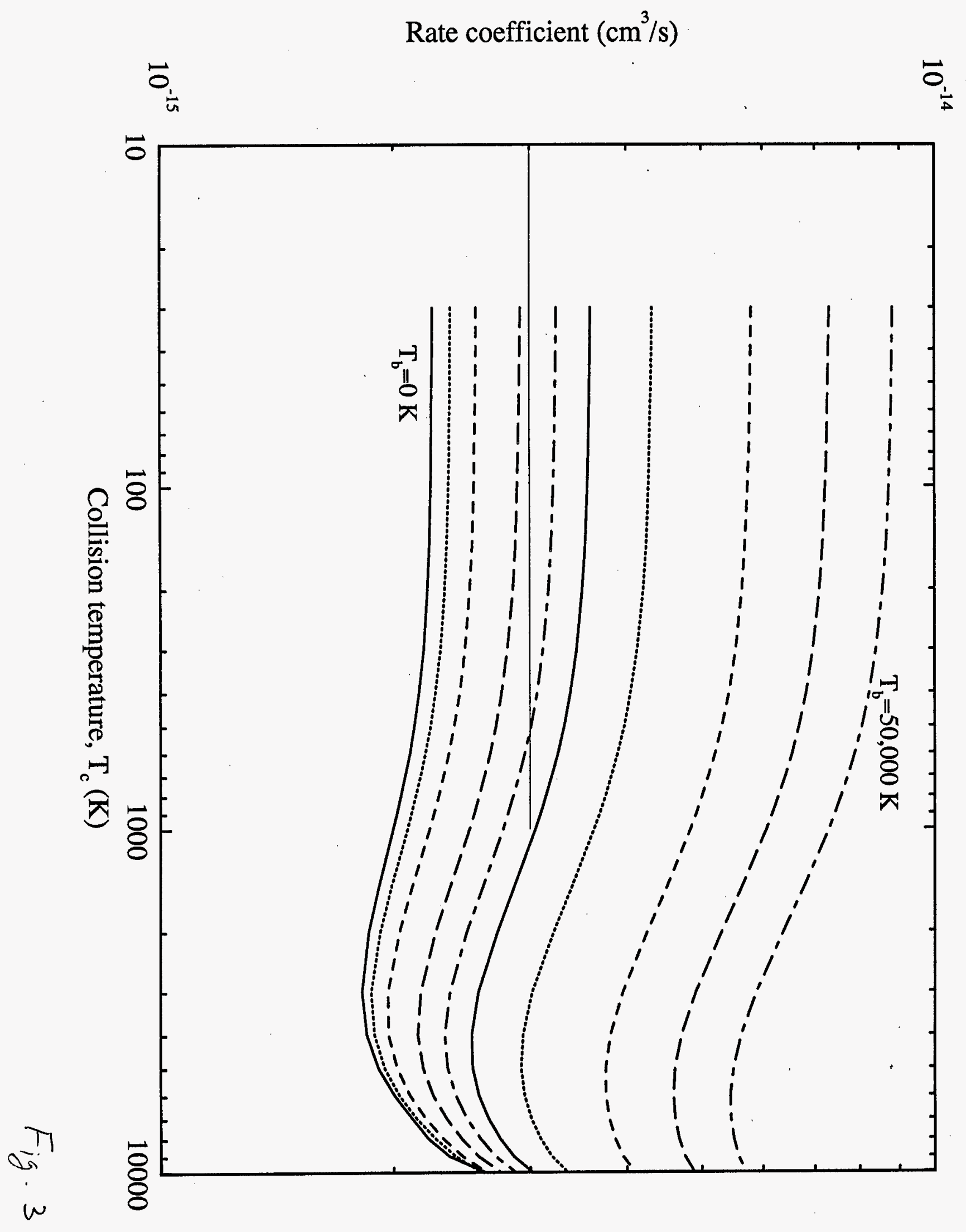




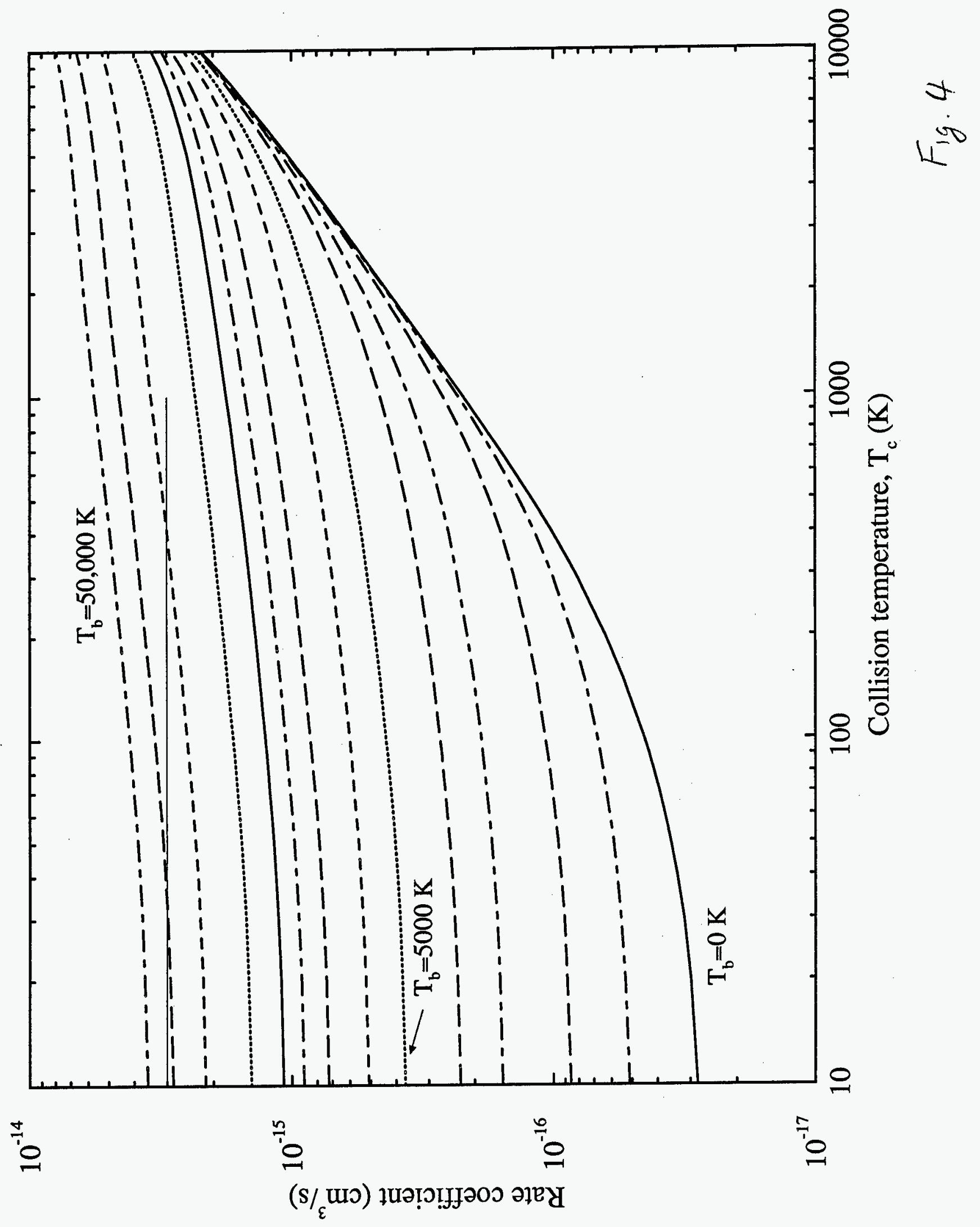




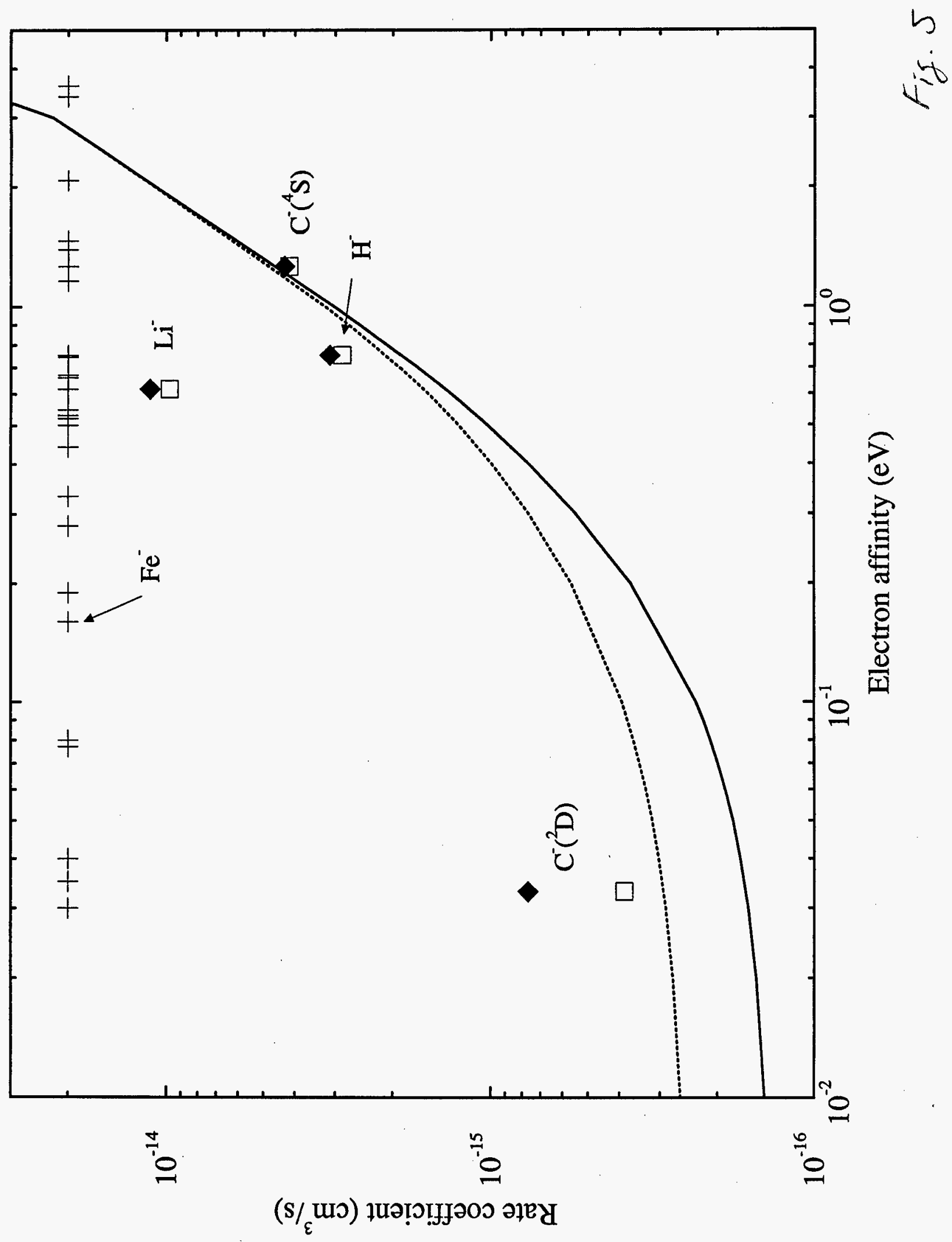


M98003152

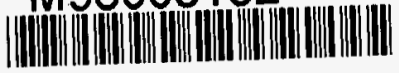

Report Number (14) ORNL/CP-96202
CONE-980417--

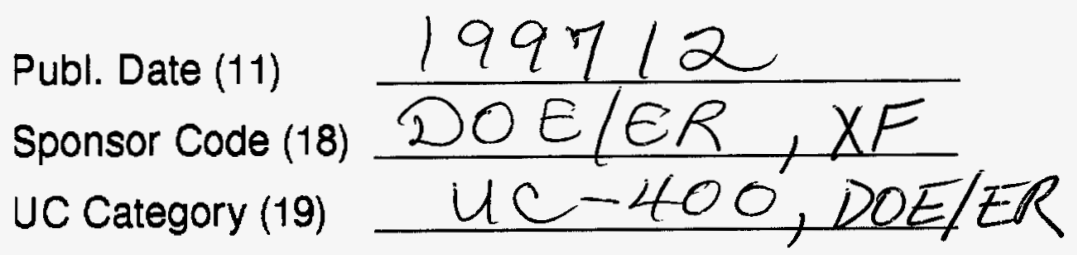

DOE 